\title{
In Situ Observation of Structural Changes in Low-dimensional Materials by Means of TEM and STEM
}

\author{
Kazu Suenaga
}

AIST, Tsukuba, Ibaraki, Japan

Molecular motion and/or defect formation can be introduced by the electron beam during TEM/STEM observations. Direct imaging of structural changes and chemical reactions for individual molecules encapsulated in carbon nanotubes was first reported in 2001 [1]. Detailed process of chemical reactions involving atomic migration was then clearly observed in 2004 [2]. Since then, a variety of molecular motions and chemical reactions happening under the electron beam were reported especially in carbon nanotubes as a test tube. We reported motions of functionalized fullerene molecules [3, 4], atomic migrations of metallofullerenes [5], and their alkali or halide complexes [6, 7]. Encapsulation of molecules in a confined nano-space, such as inside nanotubes, is a great way to prevent from some of the electron beam damages. First, the steric hindrance does not allow the neighboring molecules be present and the cross-links of broken bonds cannot be then abundant. Also the isolation of molecules from the ambient residual gas in the TEM columns is quite effective to diminish the chemical etching, which is supposedly happening with the interaction of ionized gas molecules under the electron beam.

Atomic defects or edge structures are important in crystalline materials, especially in low-dimensional materials, since the interrupted periodicities strongly affect their physical and/or chemical properties. In bulk crystals, electron microscopes have been widely used to examine structural defects such as dislocations and grain boundaries, which are regarded as one- and two-dimensional structural defects, respectively. In contrast, individual point defects (zero-dimensional defects such as mono-vacancies, impurity/dopant atoms) were believed to be difficult to investigate, with both atomic sensitivity and atomic resolution required in the analytical techniques employed. In addition, the specimens need to be very thin in order to detect the individual point defects from image contrast in TEM. After a monovacancy was first observed by TEM and proved to be stable even in low-dimensional carbon structures [8], studies of point defects in mono-layered materials had become very popular among scientists. Vacancies and topological defects in graphene were commonly examined at atomic level $[9,10,11]$.

Choice of the accelerating voltage is important for observations of such structural changes. High acceleration voltage induces much massive structural damages which is often used to observe thick bulk materials. On the other hand, not a high accelerating voltage which maybe slightly above the damage threshold would be ideal for the detailed observation of atomic process during the in situ TEM/STEM experiments. Especially, observation of small molecules made of light elements does require reducing the accelerating voltage as low as possible, in order not to destroy the molecular structures by the knock-on effect and to enhance the image/EELS contrast. In order to compensate the reduced spatial resolution due to the lower accelerating voltage, more sophisticated electron optics are definitively required to reduce the residual geometric/chromatic aberrations [12].

Another important factor is the base pressure of vacuum in the TEM column. Most of the damage is not simply regarded as the pure knock-on effect, but the chemical etching effect due to the residual gas in the column play a significant role when the atomic defects are induced. One must monitor the partial gas pressures of the residual gases in the column to fully understand the mechanism of electron beam damages [13]. In this presentation, we will show some examples how the structural changes are happening under TEM/STEM observations [14]. 


\section{References}

[1] T. Okazaki et al., J. Am. Chem. Soc., 2001, 123, 9673-9674

[2] K. Urita et al., NanoLett., 2004,4, 2451-2454

[3] Z. Liu et al., Phys. Rev. Lett. 2004, 96, 088304

[4] Z. Liu et al., Nature Nanotech., 2007, 2, 422-425

[5] Y. Sato et al., Phys. Rev. B 73, 233409, 2006

[6] L. Guan et al., Phys. Rev. Lett., 94, 045502, 2005

[7] L. Guan et al., J. Am. Chem. Soc., 2007 129, 8954-8955

[8] A. Hashimoto et al., Nature, 430 (2004) pp.870-873

[9] K. Suenaga et al., Nature Nanotech., 2 (2007) pp.358-360

[10] J. Meyer et al., Nano lett., 8 (2008) pp.3582

[11] C. O. Girit, et al., Science 323, 1705-1708 (2009).

[12] T. Sasaki et al., J. Electron Microsc. 59, s7-s13 (2010).

[13] S. Morishita et al., this meeting.

[14] The work is partially supported by JST Research Acceleration Programme. 